\title{
The 12/15-lipoxygenase pathway counteracts fibroblast activation and experimental fibrosis
}

\author{
Gerhard Krönke, ${ }^{1,2}$ Nicole Reich, ${ }^{1}$ Carina Scholtysek, ${ }^{1,2}$ Alfiya Akhmetshina, ${ }^{1}$ \\ Stefan Uderhardt, ${ }^{1,2}$ Pawel Zerr, ${ }^{1}$ Katrin Palumbo, ${ }^{1}$ Veronika Lang, ${ }^{1}$ Clara Dees, ${ }^{1}$ \\ Oliver Distler, ${ }^{3}$ Georg Schett, ${ }^{1}$ Jörg H W Distler ${ }^{1}$
}

'Department of Internal Medicine III and Institute for Clinical Immunology, University of Erlangen-Nuremberg,

Erlangen, Germany

${ }^{2}$ Nikolaus Fiebiger Center of

Molecular Medicine, University

Hospital Erlangen, University of

Erlangen-Nuremberg, Erlangen,

Germany

${ }^{3}$ Center of Experimental

Rheumatology and Zurich Center of Integrative Human

Physiology, University Hospital Zurich, Zurich, Switzerland

\section{Correspondence to}

Jörg H W Distler, Department of Internal Medicine III and Institute for Clinical Immunology, University of Erlangen-Nuremberg, Erlangen D-91054, Germany;

joerg.distler@uk-erlangen.de

GK and NR contributed equally to the manuscript.

Received 8 September 2011 Accepted 16 December 2011 Published Online First 20 January 2012

\begin{abstract}
Background Idiopathic and inflammation-dependent fibrotic diseases such systemic sclerosis (SSc) impose a major burden on modern societies. Understanding endogenous mechanisms, which counteract fibrosis, may yield new therapeutic approaches. Lipoxins are highly potent lipid mediators, which have recently been found to be decreased in SSc.

Objectives To determine the potential role of 12/15-lipoxygenase (12/15-LO), the key enzyme for the synthesis of lipoxins, in fibrosis.

Methods Two mouse models for experimental dermal fibrosis (bleomycin-induced dermal fibrosis and tight-skin 1 mouse model) together with bone marrow transfers were used in wildtype and 12/15- $\mathrm{LO}^{-/-}$mice to elucidate the role of this enzyme during dermal fibrosis. Primary dermal fibroblasts of wildtype and 12/15- $\mathrm{LO}^{-/-}$mice, and 12/15-LO-derived eicosanoids, were used to identify underlying molecular mechanisms
\end{abstract}

Results In both models, 12/15-LO $\%$ mice exhibited a significant exacerbation of the fibrotic tissue response. Bone marrow transfer experiments disclosed a predominant role of mesenchymal cell-derived 12/15-L0 in these antifibrotic effects. Indeed, 12/15- $\mathrm{LO}^{-/}$fibroblasts showed an enhanced activation of the mitogen-activated protein-kinase pathway and an increased col 1a2 mRNA expression in response to stimulation with transforming growth factor $\beta$ (TGF $\beta$ ), whereas 12/15-L0-derived eicosanoids blocked these TGF $\beta$-induced effects.

Conclusions These data indicate that $12 / 15-\mathrm{LO}$ and its metabolites have a prominent antifibrotic role during dermal fibrosis. This opens new opportunities for therapeutic approaches in the treatment of fibrotic diseases.

\section{INTRODUCTION}

Systemic sclerosis (SSc) is a rare connective tissue disease with unknown aetiology that affects the skin and a variety of internal organs, including the lungs, the gastrointestinal tract and the heart. ${ }^{1}$ Histopathological hallmarks of early stages of SSc are perivascular inflammatory infiltrates and a reduced capillary density. Later stages of the disease are characterised by an excessive accumulation of extracellular matrix (ECM) components. ${ }^{2}$ The resulting fibrosis is a major cause of death in $\mathrm{SSc}$ as it often results in dysfunction of the affected organs. The enhanced production of ECM components in patients with SSc is mediated by activated fibroblasts, which produce increased amounts of
ECM. ${ }^{1}$ However, the molecular mechanisms of fibroblast activation and potential counter-regulatory mechanisms, which limit the inflammatory reaction and the consecutive ECM accumulation, are incompletely understood.

Eicosanoids, such as prostaglandins, leukotrienes and lipoxins, have been shown to act as key modulators of inflammation and the following phases of resolution and tissue repair. ${ }^{3}$ During the initial phase of the inflammatory response cyclooxygenase-2 and 5-lipoxygenase (5-LO) catalyse the generation of prostaglandins and leukotrienes. While leukotrienes, primarily exert proinflammatory signals, prostaglandins have been shown to act in a proinflammatory and anti-inflammatory manner, depending on the context. Other arachidonic acid-metabolising enzymes, including human 15-lipoxygenase-1 (15-LO) and its murine orthologue the 12/15-lipoxygenase (12/15-LO), are expressed in the final stage of inflammation. These enzymes have been implicated in the generation of a subclass of lipid mediators, including lipoxin $\mathrm{A}_{4}\left(\mathrm{LXA}_{4}\right)$, which initiate the resolution phase of inflammation and exert potent anti-inflammatory effects. ${ }^{3-5}$ These different classes of eicosanoids have been also implicated in the regulation of postinflammatory tissue repair, which involves regulation of ECM production and fibrotic tissue response. ${ }^{67}$ Of particular interest, studies have demonstrated a reduction of lipoxins in the bronchoalveolar lavage of patients with SSc compared with controls, 89 indicating that lipoxins and 12/15-LO may have a role in the pathogenesis of SSc. Nevertheless, the molecular role of lipoxins and 12/15-LO in SSc has not been analysed so far.

In this study, we aimed to elucidate the role of 12/15-LO in dermal fibrosis. Using 12/15-LOdeficient mice, the bleomycin-induced model of skin-fibrosis and bone marrow transplantations, we demonstrate a protective role of this enzyme during inflammation-induced skin fibroses, which correlates with production of $\mathrm{LXA}_{4}$. 12/15-LO expression in mesenchymal, but not in haematopoietic cells, was required for this protective effect. By crossing $12 / 15-\mathrm{LO}^{-/}$mice into the tight-skin-1 (tsk-1) background, we confirmed a direct antifibrotic and protective role of 12/15-LO during tissue fibrosis in vivo. The following in vitro analysis of 12/15-LO-/- fibroblasts showed an enhanced activation state, including an aberrant activation of the mitogen-activated protein (MAP)-kinase pathway 
A
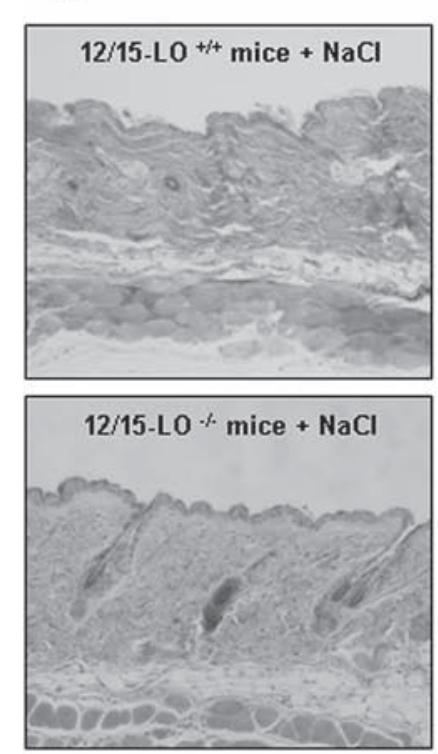

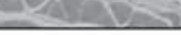
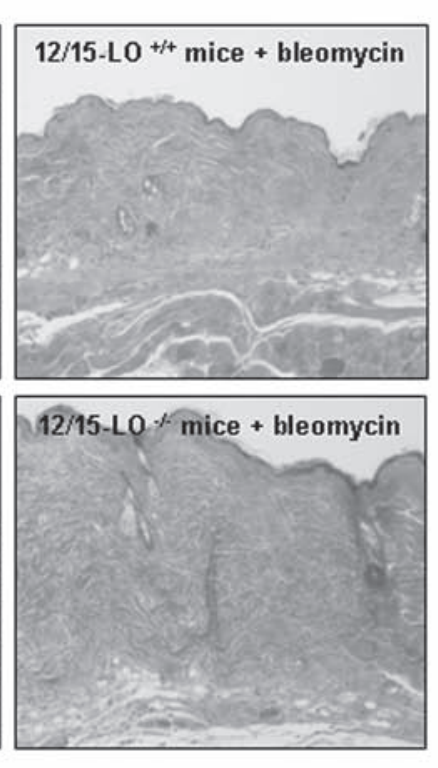

B
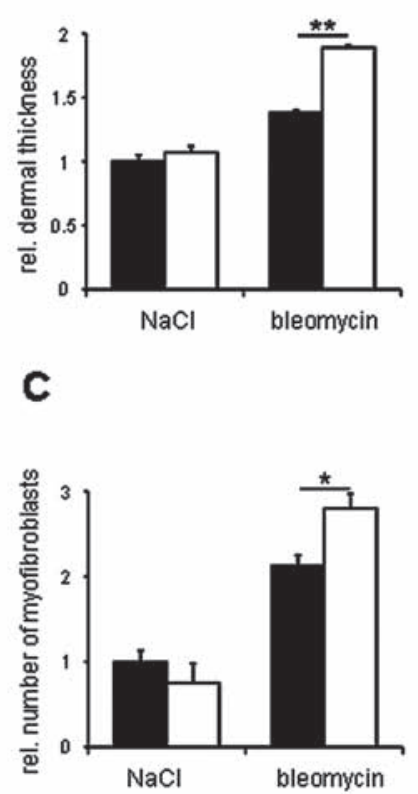

$12 / 15-\mathrm{LO}^{+/ 4+}$

$12 / 15-\mathrm{LO} \%$

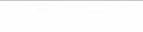

D
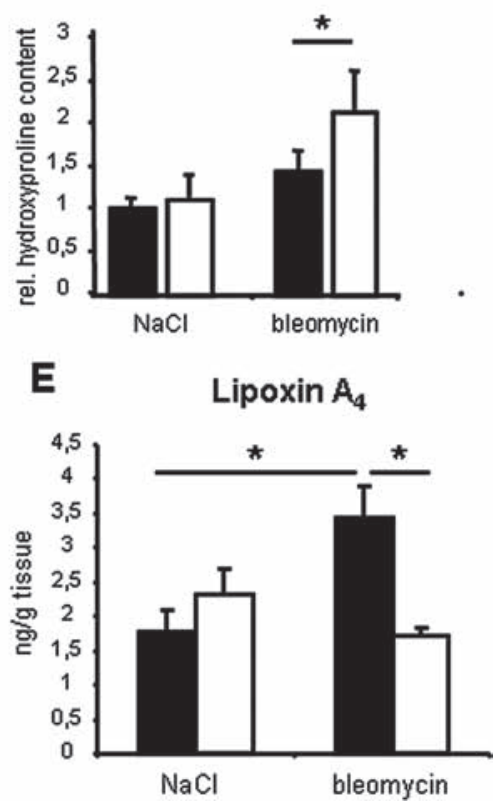

Figure 1 12/15-Lipoxygenase (12/15-L0) deficiency increases the susceptibility to bleomycin-induced dermal fibrosis. Representative skin sections (stained with haematoxylin and eosin, $x 100)(A)$, quantification of dermal thickness (B), of myofibroblast content (C) and of hydroxyproline content (D) after histological analysis of the skin of $12 / 15-\mathrm{LO}^{+/+}$and $12 / 15-\mathrm{LO}^{-/}$mice, which were treated with $\mathrm{NaCl}$ and bleomycin, respectively. (E) Results of the measurement of the lipoxin $\mathrm{A}_{4}$ content by competitive ELISA. The levels of $\mathrm{NaCl}$-treated $12 / 15-\mathrm{LO}{ }^{+/+}$mice were defined as 1 ; other results were normalised to this value. Data represent the mean \pm SEM. ${ }^{*} p<0.05 ;{ }^{* *} p<0.01$.

and overwhelming production of collagen in the absence of 12/15-LO. Conversely, addition of different 12/15-LO-derived eicosanoids, including LXA4, reversed this phenotype.

\section{MATERIAL AND METHODS}

\section{Bleomycin-induced dermal fibrosis in 12/15-LO-deficient mice}

Mice deficient for 12/15-LO $\left(12 / 15-\mathrm{LO}^{-/}\right)$have been described earlier ${ }^{10}$ and were purchased from the Jackson Laboratory (Bar Harbor, Maine, USA). 12/15- $\mathrm{LO}^{-/}$mice were maintained on a C57Bl/6 background. Matched wildtype C57Bl/6 mice expressing $12 / 15-\mathrm{LO}\left(12 / 15-\mathrm{LO}^{+/+}\right)$from the same breeding groups were used as controls. Skin fibrosis was induced in 6-week-old mice by local injections of bleomycin for 4 weeks as described. ${ }^{11} 12$ Briefly, $100 \mu \mathrm{l}$ of bleomycin dissolved in $0.9 \%$ sodium chloride $(\mathrm{NaCl})$ at a concentration of $0.5 \mathrm{mg} / \mathrm{ml}$ was administered every other day by subcutaneous injections in defined areas of $1 \mathrm{~cm}^{2}$ at the upper back. Subcutaneous injections of $100 \mu 10.9 \% \mathrm{NaCl}$ were used as controls. Four different groups, two consisting of $12 / 15-\mathrm{LO}^{-/-}$mice and two of $12 / 15-\mathrm{LO}^{+/+}$mice, were analysed. One group of $12 / 15-\mathrm{LO}^{-/}$mice and one group of $12 / 15-\mathrm{LO}^{+/+}$ mice were challenged with bleomycin, whereas the other groups were injected with $\mathrm{NaCl}$. Mice were killed by cervical dislocation after 4 weeks. Each group consisted of six to eight mice.

\section{Bone marrow transplantation}

To investigate the relative contribution of fibroblasts and other mesenchymal cells, on the one hand, and bone marrow-derived cells, on the other, to the phenotype of $12 / 15-\mathrm{LO}^{-/}$mice, bone marrow transplantation experiments were performed as described.$^{13}$ Female $12 / 15-\mathrm{LO}^{-/}$and $12 / 15-\mathrm{LO}^{+/+}$mice served as donors for bone marrow. For isolation of bone marrow cells, tibial and femur bones were prepared under sterile conditions. Bone marrow cells were flushed from the bone marrow cavities with phosphate-buffered saline and subsequently filtered through $70 \mu \mathrm{m}$ nylon mashes (BD Biosciences, Heidelberg, Germany). After lyses of erythrocytes, the isolated bone marrow cells were transplanted without further purification or in vitro expansion. Male $12 / 15-\mathrm{LO}^{-/}$or $12 / 15-\mathrm{LO}^{+/+}$mice were transplanted at an age of 4 weeks. Twenty hours before transplantation, recipient 12/15-LO-/- or $12 / 15-\mathrm{LO}^{+/+}$mice underwent whole-body irradiation with $11 \mathrm{~Gy}$. For transplantation, $2.0 \times 10^{6}$ bone marrow cells were injected into the tail veins. Two weeks after bone marrow transplantation and after confirmation of a complete reconstitution of the haematopoiesis, mice were challenged with bleomycin for 4 weeks as described above. The different experimental groups consisted of six to nine animals each.

\section{2/15-LO deficiency in tight-skin 1 mice}

In addition to the mouse model of bleomycin-induced dermal fibrosis, the tsk-1 mouse model was used to evaluate the effects of 12/15-LO deficiency on fibrosis. The tsk-1 phenotype is caused by a dominant mutation of the fibrillin-1 gene. Mice carrying one mutated fibrillin- 1 gene (FBN1 $1^{\text {tsk-1/wildtype }}$ ) are characterised by tethered skin with accumulation of collagen in the hypodermis and hypodermal thickening. ${ }^{14}$ Tsk-1 mice were crossed with $12 / 15-\mathrm{LO}^{-/}$mice. The resulting F1 generations were backcrossed with $12 / 15-\mathrm{LO}^{+/+}$or $12 / 15-\mathrm{LO}^{-/}$mice. The F2 generations were killed at an age of 10 weeks to analyse the hypodermal thickness and the number of myofibroblasts.

\section{Histological analysis}

The dermal thickness was analysed at 100 -fold magnification by measuring the distance between the epidermal—dermal junction 
A
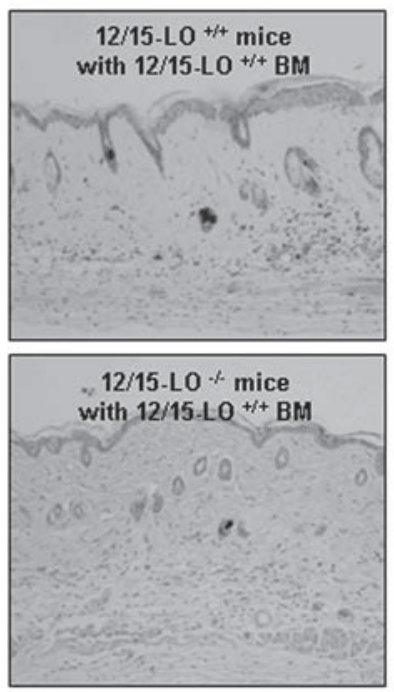
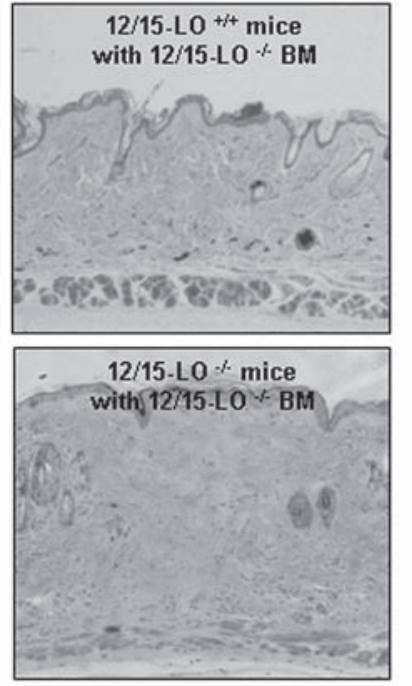

B

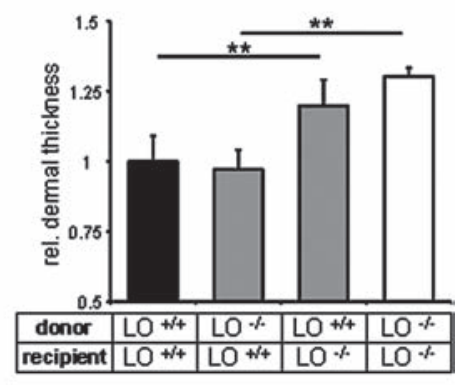

C

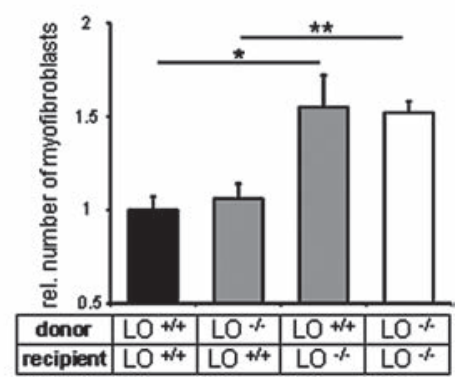

Figure 2 Absence of 12/15-lipoxygenase (12/15-LO) in resident mesenchymal cells exacerbates experimental fibrosis. Representative skin sections (stained with haematoxylin and eosin, $x 100)(A)$, quantification of dermal thickness $(B)$ and of myofibroblast content $(C)$ after histological analysis of the skin of $12 / 15-\mathrm{LO}^{+/+}$and $12 / 15-\mathrm{LO}^{-}$mice after indicated bone marrow $(\mathrm{BM})$ transplantation and treatment with $\mathrm{NaCl}$ and bleomycin, respectively. The levels of bleomycin-treated $12 / 15-\mathrm{LO}^{+/+}$mice transplanted with bone marrow (BM) from wildtype mice were defined as 1 ; other results were normalised to this value. Data represent the mean \pm SEM. ${ }^{*} \mathrm{p}<0.05 ;{ }^{* *} \mathrm{p}<0.01$.

and the dermal-subcutaneous fat junction at three sites from lesional skin of each mouse as described. ${ }^{13} 1516$ Collagen fibres were visualised by Masson's trichrome staining (Sigma-Aldrich, Munich, Germany) and analysed at 1000-fold magnification. Images were captured with a Nikon Eclipse 80i microscope (Badhoevedorp, Netherlands) equipped with a DSP 3CCD camera (Sony, Tokyo, Japan).

\section{Detection of myofibroblasts}

Myofibroblasts were identified by immunohistochemistry for $\alpha$-smooth muscle actin as described. ${ }^{17} 18$

\section{Cell culture}

Isolation and cultivation of murine dermal fibroblasts was performed as previously described. ${ }^{19}$

\section{Lipoxin $\mathrm{A}_{4}$ measurement}

Competitive ELISA techniques were used to determine levels of LXA $_{4}$ (Oxford Biomedical Research Inc), 13-S-HODE and PGE2 (both kits from assay designs). Tissue samples were homogenised and the respective eicosanoids were isolated according to the protocol provided by the manufacturer using solid-phase columns.

\section{Eicosanoids}

12-HETE and 15-HETE (Cayman Chemicals, Ann Arbor, MI, USA) were used at a concentration of $100 \mathrm{nmol} / \mathrm{l}$, 13-HODE (Cayman Chemicals) at a concentration of $5 \mu \mathrm{mol} / 1$ and $\mathrm{LXA}_{4}$ (Sigma, Munich, Germany) at $500 \mathrm{nmol} / \mathrm{l}$.

\section{Quantitative real-time PCR}

Reverse transcription (RT)-PCR was performed as previously described. ${ }^{20}$ RNA was isolated using TRIZOL reagent(Invitrogen,
Darmstadt, Germany). Total RNA (900 ng) was reverse transcribed with human leukaemia virus reverse transcriptase using the Gene Amp RNA PCR kit (Applied Biosystems, Foster City, California, USA) and oligo(dT) primers. mRNA levels were normalised to $\beta$-actin expression. The following primer sequences were used:

\section{colla1 fwd colla1 rev colla2 fwd col1a2 rev \\ GAA GCA CGT CTG GTT TGG A ACT CGA ACG GGA ATC CAT C CCA ACA AGC ATG TCT GGT TAG GA TCA AAC TGG CTG CCA CCA T}

\section{Western Blot analysis}

Cells and tissue were homogenised and lysed in Laemmli buffer. Protein concentration was determined using an RC/DC protein quantification kit (Biorad, Hercules, California, USA). Proteins were separated by electrophoresis in $12 \%$ sodium dodecyl sulphate-polyacrylamide gels. Proteins were blotted onto polyvinylidene difluoride membranes and, after blocking with $5 \%$ dry milk $/ 0.1 \%$ Tween 20 , incubated with primary and secondary peroxidase-conjugated antibodies and detected by chemiluminescence.

\section{Statistics}

Data are expressed as mean \pm SE of the mean. The Mann-Whitney $\mathrm{U}$ test was used for statistical analyses. A p value $<0.05$ was considered statistically significant.

\section{RESULTS}

12/15-LO deficiency increases the susceptibility to bleomycin-induced dermal fibrosis

To investigate the role of $12 / 15$-LO in dermal fibrosis we used the bleomycin model of skin fibrosis. Skin architecture and dermal thickness did not differ between $12 / 15-\mathrm{LO}^{-/-}$mice and 
A
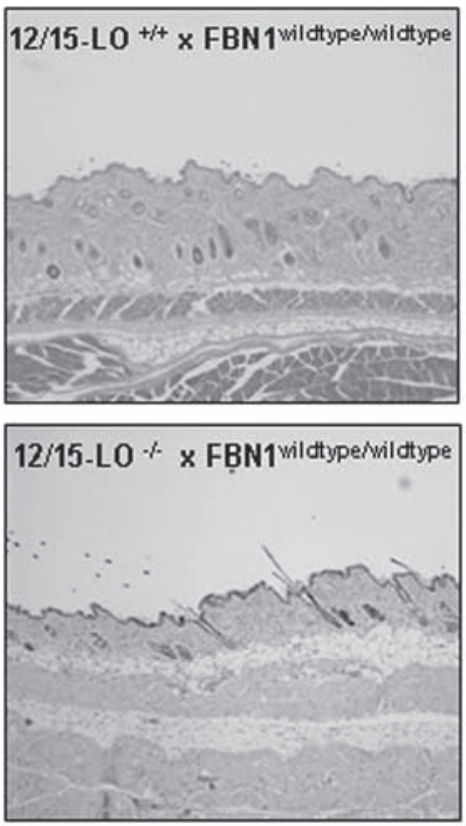
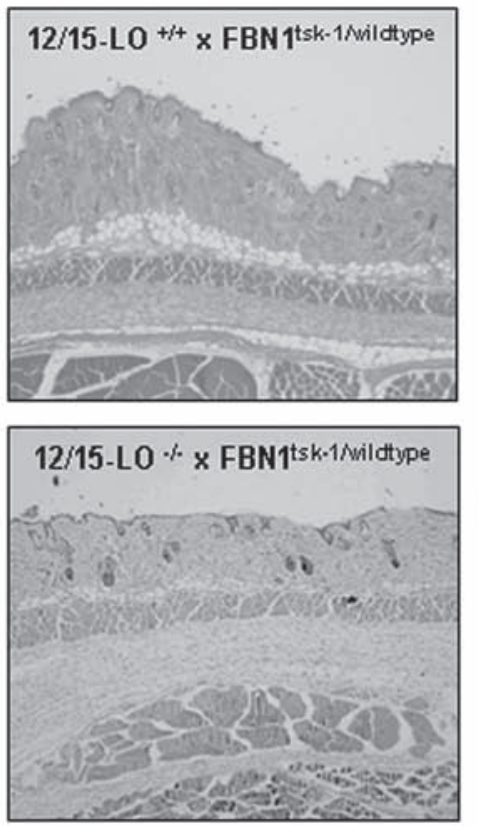

B

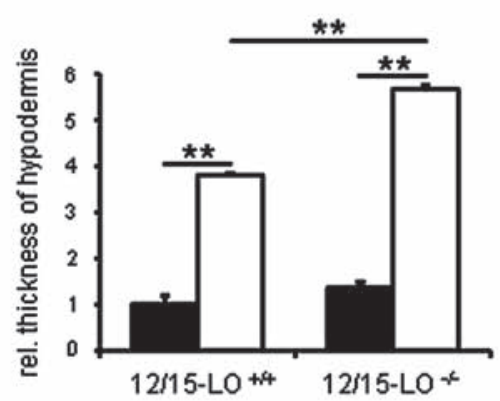

FBN1 wildtypeAwildtype

C

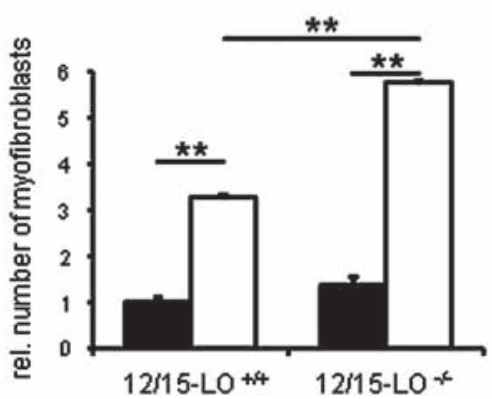

Figure 3 12/15-Lipoxygenase (12/15-LO) deficiency augments fibrosis in tight-skin-1 (tsk-1) mice. Representative skin sections (stained with haematoxylin and eosin, $x 40)(A)$, quantification of dermal thickness (B) and of myofibroblast content (C) after histological analysis of the skin of

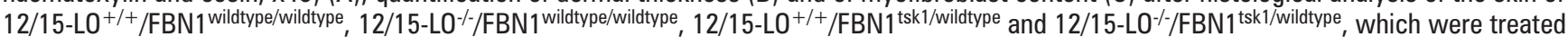
with $\mathrm{NaCl}$ and bleomycin, respectively. FBN, fibrillin-1 gene. The levels of $12 / 15-\mathrm{LO}{ }^{+/+} / \mathrm{FBN} 1$ wildtype/wildtype mice were defined as 1 ; other results were normalized to this value. Data represent the mean \pm SEM. ${ }^{*} \mathrm{p}<0.05 ;{ }^{* *} \mathrm{p}<0.01$.

$12 / 15-\mathrm{LO}^{+/+}$mice injected with $\mathrm{NaCl}$, suggesting that the skin phenotype is not generally altered in $12 / 15-\mathrm{LO}^{-/}$mice (figure $1 \mathrm{~A}, \mathrm{~B})$. Upon injection of bleomycin, however, a significantly increased fibrotic response was seen in $12 / 15-\mathrm{LO}^{-/-}$mice. The dermal thickness increased by $90 \pm 2 \%$ in $12 / 15-\mathrm{LO}^{-/-}$mice compared with $42 \pm 4 \%$ in $12 / 15-\mathrm{LO}^{+/+}$mice $(\mathrm{p}=0.001)$ (figure $\left.1 \mathrm{~A}, \mathrm{~B}\right)$.

Consistent with the increased dermal thickness, the number of myofibroblasts upon challenge with bleomycin was significantly higher in $12 / 15-\mathrm{LO}^{-/-}$mice than in $12 / 15-\mathrm{LO}^{+/+}$mice $(p=0.022)$ (figure 1C). Moreover, the increase in hydroxyproline content upon challenge with bleomycin was significantly more pronounced in $12 / 15-\mathrm{LO}^{-/}$mice than in $12 / 15-\mathrm{LO}^{+/+}$littermates (figure 1D). These data show that deficiency of $12 / 15-\mathrm{LO}$ increases the susceptibility to bleomycin-induced fibrosis and indicates a protective and antifibrotic role of $12 / 15$-LO during bleomycin-induced fibrosis.

To further determine underlying mechanisms, we performed a measurement of the $\mathrm{LXA}_{4}$ content in the skin of $12 / 15-\mathrm{LO}^{+/+}$ and $12 / 15-\mathrm{LO}^{-/}$mice after $\mathrm{NaCl}$ and bleomycin challenge, respectively. $\mathrm{LXA}_{4}$ represents a major 12/15-LO metabolite and has been shown to exert both anti-inflammatory and antifibrotic effects in vitro. ${ }^{2122}$ While we did not observe a difference in dermal $\mathrm{LXA}_{4}$ levels after injection of $\mathrm{NaCl}, \mathrm{LXA}_{4}$ levels significantly increased after injection of bleomycin. Consistent with a central role of 12/15-LO in the generation of $\mathrm{LXA}_{4}, 12 / 15-\mathrm{LO}^{-/-}$mice displayed significantly reduced amount of $\mathrm{LXA}_{4}$ (figure $1 \mathrm{E}$ ).

\section{Absence of 12/15-LO in resident mesenchymal cells exacerbates experimental fibrosis}

The mouse model of bleomycin-induced fibrosis is an inflammation-dependent model, in which bleomycin induces the influx of leucocytes into lesional skin with secondary activation of fibroblasts by leucocyte-derived profibrotic cytokines. ${ }^{23}$ Thus, aggravated fibrosis in this model might be linked to an increased inflammatory response in the form of an enhanced activation of cells of haematopoietic origin, such as leucocytes. Alternatively, an altered responsiveness of mesenchymal fibroblasts may directly cause an enhanced fibrotic response. To determine whether the protective effects of 12/15-LO during bleomycininduced skin fibrosis are linked to 12/15-LO expression in cells of haematopoietic or of mesenchymal origin, we performed a series of bone marrow transplantations. The resulting chimeric mice were challenged with bleomycin. Bleomycin challenge of $12 / 15-\mathrm{LO}^{+/+}$mice reconstituted with bone marrow from either $12 / 15-\mathrm{LO}^{-/}$or $12 / 15-\mathrm{LO}^{+/+}$resulted in a comparable increase in dermal thickness with increases of $54 \pm 10 \%$ and $54 \pm 11 \%$, respectively, compared with $\mathrm{NaCl}$-treated mice (figure $2 \mathrm{~A}, \mathrm{~B}$ ).

In contrast, $12 / 15-\mathrm{LO}^{-/}$mice showed increased susceptibility to bleomycin-induced fibrosis regardless of whether or not they were reconstituted with bone marrow cells derived from $12 / 15-\mathrm{LO}^{+/+}$mice or from $12 / 15-\mathrm{LO}^{-/}$mice. Challenge of $12 / 15-\mathrm{LO}^{-/-}$mice reconstituted with $12 / 15-\mathrm{LO}^{+/+}$bone marrow with bleomycin resulted in a $90 \pm 14 \%$ increase in dermal thickness. This increase did not differ from that seen in $12 / 15-\mathrm{LO}^{-/}$mice with $12 / 15-\mathrm{LO}^{-/}$bone marrow ( $\mathrm{p}=0.33$ ), but was significantly more pronounced than in $12 / 15-\mathrm{LO}^{+/+}$mice with $12 / 15-\mathrm{LO}^{+/+}$bone marrow and in $12 / 15-\mathrm{LO}^{+/+}$mice reconstituted with $12 / 15-\mathrm{LO}^{-/}$bone marrow $(\mathrm{p}=0.002$ and $\mathrm{p}=0.001$, respectively) (figure $2 \mathrm{~A}, \mathrm{~B}$ ). The increased differentiation of resting fibroblasts into metabolically active myofibroblasts in $12 / 15-\mathrm{LO}^{-/-}$mice was also seen in $12 / 15-\mathrm{LO}^{-/-}$mice transplanted with $12 / 15-\mathrm{LO}^{+/+}$bone marrow, whereas the myofibroblast 
A
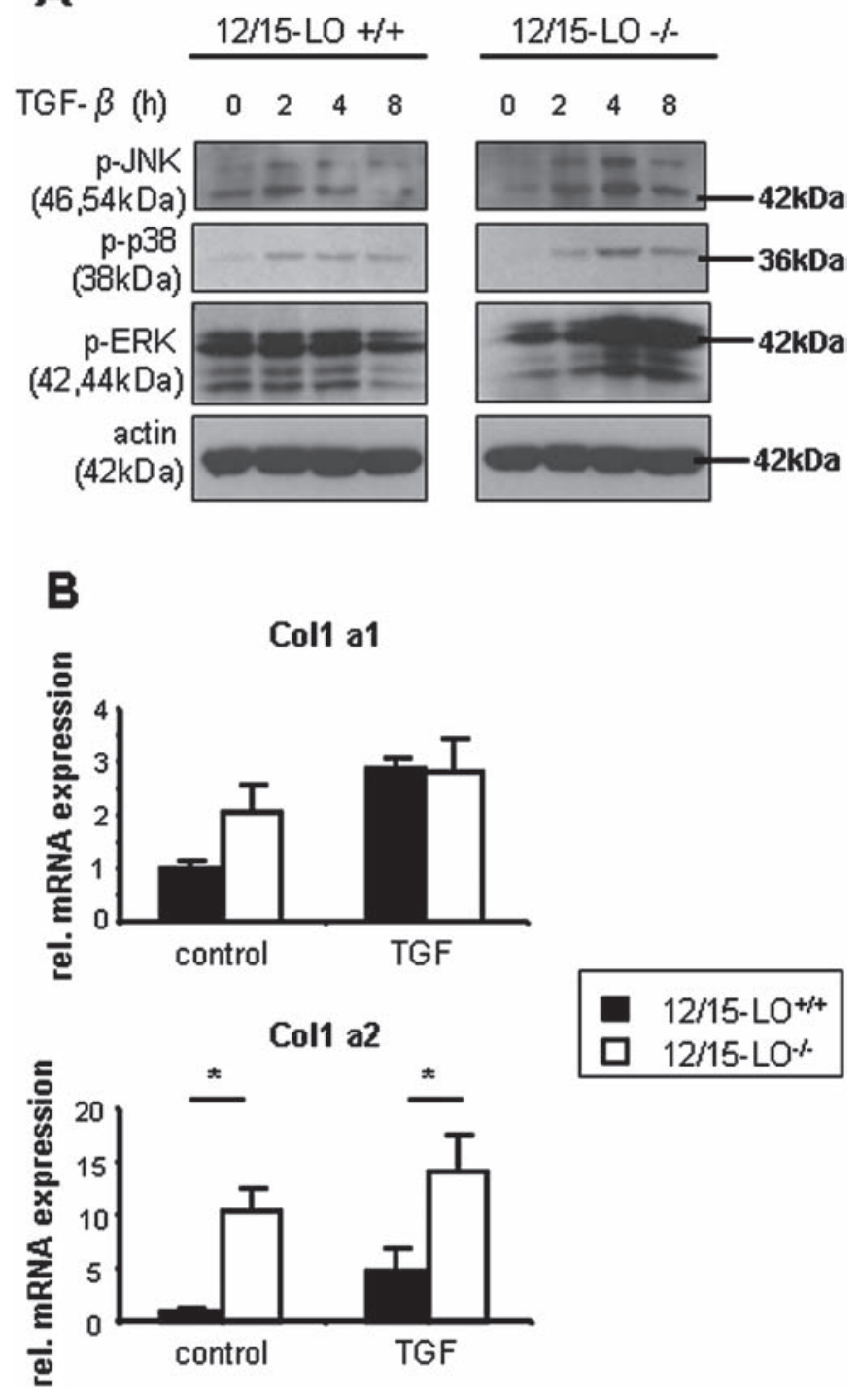

Figure 4 12/15-Lipoxygenase (12/15-LO)-deficient fibroblasts show an enhanced response to transforming growth factor $\beta$ (TGF $\beta$ ). (A) Primary dermal fibroblasts of $12 / 15-\mathrm{LO}^{+/+}$and $12 / 15-\mathrm{LO} /-$ mice were stimulated with TGF $\beta(10 \mathrm{ng} / \mathrm{ml})$ for the indicated times and phosphorylation of the indicated mitogen-activated protein (MAP)kinase pathways was analysed by Western Blot. (B) After stimulation of fibroblasts with TGF $\beta$ (10 ng/ml) for $8 \mathrm{~h}$, mRNA expression of col $1 \mathrm{a} 2$ was determined by real-time PCR.

counts in $12 / 15-\mathrm{LO}^{+/+}$mice transplanted with $12 / 15-\mathrm{LO}^{-/-}$bone marrow did not differ from those of $12 / 15-\mathrm{LO}^{+/+}$mice (figure 2C). Together, these data demonstrate that 12/15-LO expression in resident mesenchymal cells, rather than in haematopoietic cells, accounts for the protective effect of 12/15-LO during experimental fibrosis.

\section{2/15-LO deficiency augments fibrosis in tsk-1 mice}

To confirm a role of the 12/15-LO pathway in another model of skin fibrosis, we used the tsk-1 model. In contrast to the bleomycin-induced model, the tsk-1 model is an inflammation-independent model, in which fibrosis is mainly driven by endogenous activation of fibroblasts. ${ }^{23}$ Mice lacking 12/15-LO were therefore crossed with tsk-1 mice to obtain tsk-1 mice lacking both alleles for 12/15-LO (12/15-LO-//FBN1 ${ }^{\text {tsk1/wildtype }) . ~ 12 / 15-L O-/ / t s k-1 ~}$ mice were born in the expected Mendelian ratios and appeared completely normal at birth. Skin fibrosis was more pronounced in $12 / 15-\mathrm{LO}^{-/ /} / \mathrm{FBN}^{\text {tsk } 1 / \text { wildtype }}$ mice than in their $12 / 15-\mathrm{LO}^{+/+} /$ FBN1 ${ }^{\text {tsk1/wildtype }}$ littermates. Here, we observed a significantly increased hypodermal thickening and increased myofibroblast differentiation (figure $3 \mathrm{~A}-\mathrm{C}$ ). No differences in hypodermal thickness were seen between 12/15-LO-/-/FBN1 wildtype/ wildtype mice and $12 / 15-\mathrm{LO}^{+/+} / \mathrm{FBN1}$ wildtype/wildtype. These data confirm our previous results on the protective, antifibrotic role of $12 / 15-\mathrm{LO}$ in bleomycin-induced dermal fibrosis and suggest that the 12/15-LO pathway directly affects the response of fibroblasts, including the regulation of myofibroblast differentiation and ECM accumulation.

\section{2/15-LO-deficient fibroblasts show an enhanced response to TGF $\beta$}

To further elucidate underlying molecular mechanisms, we focused on the role of $12 / 15$ - LO during fibroblast activation. Therefore, fibroblasts derived from $12 / 15-\mathrm{LO}^{+/+}$and $12 / 15-\mathrm{LO}^{-1-}$ mice were stimulated with TGF $\beta$. MAP-kinase activation was monitored by Western Blot analysis, which showed increased activation of all three MAP-kinase pathways in the absence of 12/15-LO (figure 4A). Moreover, the analysis of the downstream collagen gene expression in the respective fibroblasts by quantitative real-time PCR showed significant differences. Fibroblasts isolated from 12/15-LO-deficient animals displayed increased expression of col 1a2 mRNA as compared with 12/15- $\mathrm{LO}^{+/+}$ control fibroblasts (figure 4B).

\section{2/15-LO-derived eicosanoids counteract TGF $\beta$-induced activation of fibroblasts}

Next, we determined the effects of different 12/15-LO-derived eicosanoids on TGF $\beta$-induced fibroblast activation. Consistent with an antifibrotic role of 12/15-LO and 12/15-LO-derived eicosanoids, the tested mediators, which included 12-HETE, 13-HODE, 15-HETE and $\mathrm{LXA}_{4}$, interfered with TGF $\beta$-induced activation of the different MAP-kinase pathways (figure $5 \mathrm{~A}, \mathrm{~B}$ ). Likewise, the increased TGF $\beta$-induced expression of collagen 1a2 mRNA in 12/15-LO-deficient fibroblasts was significantly reduced by these eicosanoids (figure $5 \mathrm{C}$ ). Together these data identify both $12 / 15$-LO and its metabolites as important regulators of fibroblast activation.

\section{DISCUSSION}

Although several molecular pathways that stimulate fibrosis have been identified over recent years, little is known about endogenous protective feedback loops limiting fibroblast activation and fibrosis. Our study provides evidence for a role of the 12/15-LO pathway as endogenous and negative regulator in experimental fibrosis. While we observed an increased formation of the $12 / 15$-LO product $\mathrm{LXA}_{4}$ in the skin of bleomycin-treated animals, absence of $12 / 15$-LO drastically reduced formation of this eicosanoid. This was paralleled by a drastic increase in the progression of dermal fibrosis. As expected, LXA 4 was not completely absent as enzymes other than 12/15-LO can account for LXA $_{4}$ production. ${ }^{3}$ These bone marrow transfer studies demonstrated that the antifibrotic effect of $12 / 15-\mathrm{LO}$ is due to expression of this enzyme in mesenchymal rather than haematopoietic cells. This highlights that fibroblasts and/or other mesenchymal cells are key players in the antifibrotic 12/15-LO pathway. In line with these results, we observed an exacerbation of fibrosis also in the tsk-1 model of dermal fibrosis, which is a model of less inflammation-dependent stages of skin fibrosis that are mainly driven by endogenous activation of fibroblasts. 
B

TGF (5h)

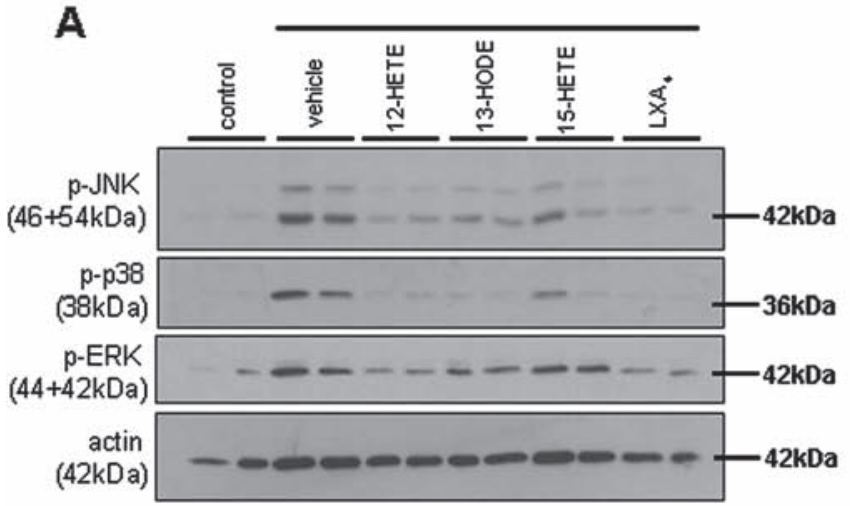

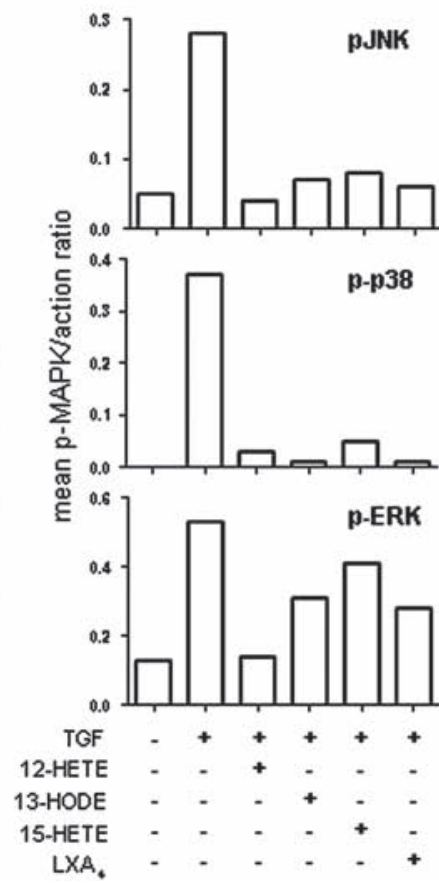

C

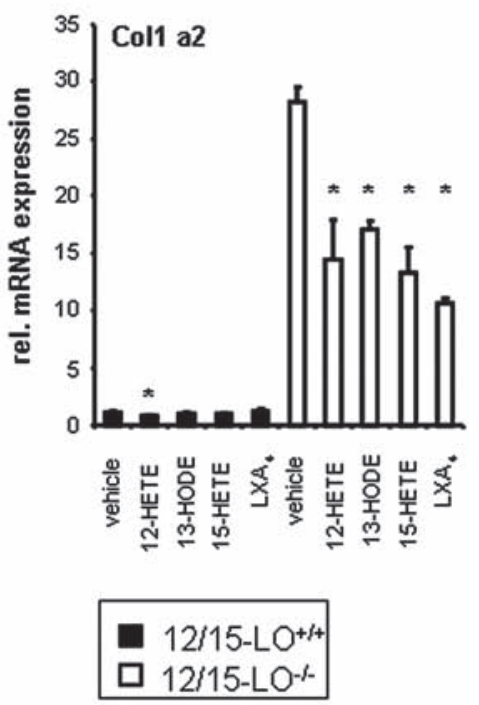

Figure 5 12/15-LO-derived eicosanoids counteract TGF $\beta$ induced activation of fibroblasts. (A) Primary dermal fibroblasts of 12/15-lipoxygenase (12/15-LO) mice were pretreated for $4 \mathrm{~h}$ with the indicated eicosanoid and stimulated with transforming growth factor $\beta$ (TGF $\beta ; 10 \mathrm{ng} / \mathrm{ml}$ ) for $5 \mathrm{~h}$ before phosphorylation of the indicated mitogen-activated protein (MAP)-kinase pathways was analysed by Western Blot. Intensities of the respective p-MAPK and their corresponding $\beta$-actin bands were measured and are depicted as mean p-MAPK/ $\beta$-actin ratios (B). (C) After $5 \mathrm{~h}$ of pretreatment with the indicated eicosanoids, fibroblasts were stimulated with TGF $\beta(10 \mathrm{ng} / \mathrm{ml})$ for $8 \mathrm{~h}$ before mRNA expression of col 1a2 was determined by real-time PCR.

Our in vitro data further confirm the important role of 12/15-LO in the response of fibroblasts to TGF $\beta$. 12/15-LOdeficient fibroblasts showed an enhanced expression of col 1a2. Moreover, 12/15-LO-deficient fibroblasts showed an aberrant signalling response to TGF $\beta$, which was characterised by an increased activation of different MAP-kinase pathways including JNK, p38 and ERK. These pathways have been previously implicated in the activation and proliferation of fibroblasts. ${ }^{24-26}$ In turn, 12/15-LO products, including 12-HETE, 13-HODE, 15-HETE and LXA 4 , were able to differentially block these intracellular signalling pathways after stimulation with TGF $\beta$ and reduce the expression of col 1a2. Together these data provide evidence for a feedback loop involving 12/15-LO and 12/15-LO-derived eicosanoids, which limits the fibrotic tissue response. The finding that a deficiency of 12/15-LO exacerbates fibrosis in bleomycin-induced dermal fibrosis and in the tsk-1 model suggests that this feedback loop may be relevant both during early, inflammatory phases and later, in the non-inflammatory stages of fibrotic diseases.

Our results are consistent with a previous report that lipoxin analogues prevent experimental pulmonary fibrosis. ${ }^{27}$ Again, these protective effects seem to be linked both to anti-inflammatory and direct antifibrotic effects of these eicosanoids, because lipoxins have been shown to block the activation of pulmonary fibroblasts in vitro. ${ }^{22}$ Interestingly, other eicosanoids such as leukotrienes clearly have opposing and profibrotic effects. ${ }^{7}$ This indicates a complex crosstalk, which controls the fibrotic tissue response on the level of arachidonic acid metabolism.

The clinical relevance of lipoxins and leukotrienes for SScassociated fibrosis is highlighted by studies on the lipid profile in the bronchoalveolar lavage of patients with SSc. Significantly decreased levels of $\mathrm{LXA}_{4}$ were measured in SSc, whereas the concentration of leukotrienes was increased. ${ }^{89}$ Together, these results suggest that a shift in the balance of these profibrotic and antifibrotic lipid mediators may contribute to the pathogenesis of SSc by stimulating fibroblast activation and progression of fibrosis.

Together our data identify the $12 / 15-\mathrm{LO}-\mathrm{LXA}_{4}$ pathway as a negative key regulator of the fibrotic tissue response, rendering it a potential target for therapeutic intervention in other fibrotic diseases. Given that lipoxins are currently being explored as new therapeutic agents for inflammatory diseases, these findings may have direct translational implications. However, further studies with exogenous lipoxins in preclinical models of fibrosis are warranted.

Acknowledgments The authors thank Maria Halter, Martin Steffen, Cornelia Stoll and Isabell Schmidt for excellent technical assistance.

Funding Grants DI 1537/1-1, DI 1537/2-1, DI 1537/4-1, AK 144/1-1 and SCHE 1583/7-1 of the Deutsche Forschungsgesellschaft, grants A20 and A40 of the IZKF in Erlangen, the ELAN-Program of the University of Erlangen-Nuremberg and the Career Support Award of Medicine of the Ernst Jung Foundation

Competing interests None.

Provenance and peer review Not commissioned; externally peer reviewed.

\section{REFERENCES}

1. Gabrielli A, Avvedimento EV, Krieg T. Scleroderma. N Engl J Med 2009;360:1989-2003

2. Abraham DJ, Krieg T, Distler J, et al. Overview of pathogenesis of systemic sclerosis. Rheumatology 2009;48:iii3-7.

3. Serhan CN, Savill J. Resolution of inflammation: the beginning programs the end. Nat Immunol 2005;6:1191-7.

4. Levy BD, Clish CB, Schmidt B, et al. Lipid mediator class switching during acute inflammation: signals in resolution. Nat Immunol 2001;2:612-9.

5. Kühn H, O'Donnell VB. Inflammation and immune regulation by 12/15-lipoxygenases. Prog Lipid Res 2006:45:334-56. 
6. Börgeson E, Docherty NG, Murphy M, et al. Lipoxin A4 and benzo-lipoxin A4 attenuate experimental renal fibrosis. FASEB J 2011;25:2967-79.

7. Ryan A, Godson C. Lipoxins: regulators of resolution. Curr Opin Pharmacol 2010;10:166-72.

8. Kowal-Bielecka 0, Kowal K, Distler 0, et al. Mechanisms of Disease: leukotrienes and lipoxins in scleroderma lung disease-insights and potential therapeutic implications. Nat Clin Pract Rheumatol 2007;3:43-51.

9. Kowal-Bielecka 0, Kowal K, Distler 0, et al. Cyclooxygenase- and lipoxygenasederived eicosanoids in bronchoalveolar lavage fluid from patients with scleroderma lung disease: an imbalance between proinflammatory and anti-inflammatory lipid mediators. Arthritis Rheum 2005;52:3783-91.

10. Sun $\mathbf{D}$, Funk $\mathrm{CD}$. Disruption of $12 / 15$-lipoxygenase expression in peritoneal macrophages. Enhanced utilization of the 5-lipoxygenase pathway and diminished oxidation of low density lipoprotein. J Biol Chem 1996:271:24055-62.

11. Akhmetshina A, Dees C, Pileckyte $\mathrm{M}$, et al. Dual inhibition of C-abl and PDGF receptor signaling by dasatinib and nilotinib for the treatment of dermal fibrosis. FASEB J 2008;22:2214-22.

12. Palumbo K, Zerr P, Tomcik M, et al. The transcription factor JunD mediates transforming growth factor $\{$ beta $\}$-induced fibroblast activation and fibrosis in systemic sclerosis. Ann Rheum Dis 2011;70:1320-6.

13. Marquart S, Zerr P, Akhmetshina A, et al. Inactivation of the cannabinoid receptor CB1 prevents leukocyte infiltration and experimental fibrosis. Arthritis Rheum 2010;62:3467-76.

14. Avouac J, Fürnrohr BG, Tomcik M, et al. Inactivation of the transcription factor STAT4 prevents inflammation-driven fibrosis in systemic sclerosis animal models. Arthritis Rheum 2011;63:800-9.

15. Dees C, Akhmetshina A, Zerr P, et al. Platelet-derived serotonin links vascular disease and tissue fibrosis. J Exp Med 2011;208:961-72.
16. Dees C, Zerr P, Tomcik M, et al. Inhibition of Notch signaling prevents experimental fibrosis and induces regression of established fibrosis. Arthritis Rheum 2011;63:1396-404.

17. Akhmetshina A, Dees C, Busch N, et al. The cannabinoid receptor CB2 exerts antifibrotic effects in experimental dermal fibrosis. Arthritis Rheum 2009;60:1129-36.

18. Akhmetshina A, Venalis $\mathrm{P}$, Dees $\mathrm{C}$, et al. Treatment with imatinib prevents fibrosis in different preclinical models of systemic sclerosis and induces regression of established fibrosis. Arthritis Rheum 2009;60:219-24.

19. Skhirtladze C, Distler O, Dees C, et al. Src kinases in systemic sclerosis: central roles in fibroblast activation and in skin fibrosis. Arthritis Rheum 2008;58:1475-84.

20. Krönke G, Bochkov VN, Huber J, et al. Oxidized phospholipids induce expression of human heme oxygenase-1 involving activation of cAMP-responsive element-binding protein. J Biol Chem 2003:278:51006-14.

21. Wu SH, Lu C, Dong L, et al. Lipoxin A4 inhibits TNF-alpha-induced production of interleukins and proliferation of rat mesangial cells. Kidney Int 2005;68:35-46.

22. Bian XW, Jiang XF, Chen JH, et al. Increased angiogenic capabilities of endothelial cells from microvessels of malignant human gliomas. Int Immunopharmacol 2006;6:90-9.

23. Beyer C, Schett G, Distler 0 , et al. Animal models of systemic sclerosis: prospects and limitations. Arthritis Rheum 2010;62:2831-44.

24. Ihn H. Autocrine TGF-beta signaling in the pathogenesis of systemic sclerosis. J Dermatol Sci 2008; 49:103-13.

25. Ihn H, Yamane K, Tamaki K. Increased phosphorylation and activation of mitogen-activated protein kinase p38 in scleroderma fibroblasts. J Invest Dermatol 2005:125:247-55.

26. Katsumoto TR, Whitfield ML, Connolly MK. The pathogenesis of systemic sclerosis. Annu Rev Pathol 2011;6:509-37.

27. Martins V Valença SS, Farias-Filho FA, et al. ATLa, an aspirin-triggered lipoxin A4 synthetic analog, prevents the inflammatory and fibrotic effects of bleomycin-induced pulmonary fibrosis. J Immunol 2009;182:5374-81. 


\section{ARD The 12/15-lipoxygenase pathway counteracts} fibroblast activation and experimental fibrosis

Gerhard Krönke, Nicole Reich, Carina Scholtysek, Alfiya Akhmetshina, Stefan Uderhardt, Pawel Zerr, Katrin Palumbo, Veronika Lang, Clara Dees, Oliver Distler, Georg Schett and Jörg H W Distler

Ann Rheum Dis 2012 71: 1081-1087 originally published online January 20, 2012

doi: 10.1136/annrheumdis-2011-200745

Updated information and services can be found at:

http://ard.bmj.com/content/71/6/1081

\section{These include:}

References This article cites 27 articles, 8 of which you can access for free at: http://ard.bmj.com/content/71/6/1081\#BIBL

Email alerting service

Receive free email alerts when new articles cite this article. Sign up in the box at the top right corner of the online article.

Topic Articles on similar topics can be found in the following collections

Collections

Immunology (including allergy) (5117)

Connective tissue disease (4234)

\section{Notes}

To request permissions go to:

http://group.bmj.com/group/rights-licensing/permissions

To order reprints go to:

http://journals.bmj.com/cgi/reprintform

To subscribe to BMJ go to:

http://group.bmj.com/subscribe/ 